A man presented with foot drop and 2 months later an infrarenal occlusion of his abdominal aorta was discovered. Initial evaluation found no structural etiology for his nerve injury. His neurologic deficits progressed until gangrenous changes developed in his feet leading to the discovery of aortic occlusion. This case demonstrates that peripheral nerve injury in the legs may signal underlying large vessel occlusive disease. We discuss the localization and potential mechanisms of ischemic nerve injury. (c) 1995 John Wiley \& Sons, Inc.

Key words: ischemic neuropathy $\bullet$ aortic occlusive disease $\bullet$ peripheral nerve $\cdot$ peripheral vascular disease

MUSCLE \& NERVE 18:899-903 1995

\title{
FOOT DROP AS A HARBINGER OF AORTIC OCCLUSION
}

\author{
WENDY L. LARSON, MD, and JOHN J. WALD, MD
}

Peripheral nerve injury may be a harbinger of underlying large vessel vascular pathology. Nerve injury secondary to ischemia has been described since the 1940s, but often is difficult to recognize clinically as a potential etiology of peripheral nerve dysfunction. ${ }^{1}$ The majority of case reports describing peripheral nerve or spinal cord injury due to vascular pathology in the aorta relate to acute complications of aortic aneurysms or repair of aortoiliac occlusive disease..$^{2,4,5,11}$ In these cases, the diagnosis of nerve injury came after identification of the vascular pathology, and frequently involved surgical manipulation of the vessel. The precise localization of nerve injury in this setting may be difficult by neurologic exam alone and usually requires the aid of electromyography.

In this report, we summarize the clinical, electrophysiologic, radiologic, and pathologic findings in a patient with lumbosacral plexopathy due to aortic occlusive disease, whose neurologic deficits preceded identification of the vascular lesion. The presumed pathophysiology and anatomic localization of ischemic peripheral nerve injury will be discussed.

\section{CASE REPORT}

Clinical Mistory. A 41-year-old man was seen for evaluation of sudden worsening of lower extremity

\footnotetext{
From the Department of Neurology, University of Michigan Medical Center, and Veterans Administration Hospital, Ann Arbor, Michigan.

Address reprint requests to $\mathrm{Dr}$. Wendy $\mathrm{L}$. Larson, University of Michigan Hospitals, 1500 E. Medical Center Drive, Ann Arbor, MI 48109

Accepted for publication February 26, 1995

CCC 0148-639X/95/080899-05

(C) 1995 John Wiley \& Sons, Inc
}

weakness and numbness, coincident with the development of ischemic changes in the distal right leg.

The patient was well until late August of 1993, when he noted persistent crampy pain in his left buttock and calf, worsened by activity. In late September, when rising from a bar stool, he experienced the sudden onset of numbness along the lateral aspect of his left leg and "flapping" of his left foot on the floor when he attempted to walk. The weakness in his left foot lasted 2-3 h and then resolved. The following day he developed similar symptoms after a prolonged period of sitting, and the weakness of the foot persisted.

Upon initial medical evaluation, he reported a history of hypertension and tobacco use. He denied back injury, right leg weakness, or bladder dysfunction. On examination, he was afebrile and normotensive, with weakness of the left lower extremity in the distribution of the peroneal nerve, diminished pin prick in the L-5-S-1 dermatome, and a depressed left ankle jerk.

Normal studies during the initial work-up included a complete blood count, electrolyte and chemistry profile, serum protein electrophoresis, creatinine phosphokinase, VDRL, antinuclear antibody, carcinoembryonic antigen, prostate specific antigen, and electrocardiogram. Lumbar puncture was normal with a protein of 41 , glucose of 68 , and one white blood cell. Sedimentation rate was elevated at 38 , cholesterol was elevated at 257 . EMG was remarkable for abnormal spontaneous activity in the left anterior tibialis and medial gastrocnemius, in addition to the left lower lumbar paraspinous muscles (Table 1). Interpretation of the study was of an early L-5 radiculopathy. Evaluation for underlying structural disease in the lum- 


\begin{tabular}{|c|c|c|c|c|c|c|}
\hline & \multicolumn{3}{|c|}{ Initial study $(9 / 93)$} & \multicolumn{3}{|c|}{ Follow-up study (2/94) } \\
\hline & Right & Left & Normal & Right & Left & Normal \\
\hline Tibial & & & Not provided & & & \\
\hline $\begin{array}{l}\text { Amplitude } \\
\text { Distal latency }\end{array}$ & $">5 "$ & $">5 "$ & & NR & NR & \\
\hline Conduction velocity & 44 & 42 & & & & \\
\hline F-wave latency & 52 & 56 & & & & \\
\hline \multicolumn{7}{|l|}{ Peroneal } \\
\hline Amplitude & $">5 "$ & $">5 "$ & & NR & 0.2 & $>2$ \\
\hline Distal latency & & & & & 3.5 & $<6.1$ \\
\hline Conduction velocity & 45 & 44 & & & 32.3 & $>41$ \\
\hline F-wave latency & 54 & NR & & & $N R$ & \\
\hline \multicolumn{7}{|l|}{ Sural } \\
\hline $\begin{array}{l}\text { Amplitude } \\
\text { Distal latency }\end{array}$ & 10 & 15 & & NR & NR & \\
\hline$H$ reflex & 30.8 & 33.6 & & ND & ND & \\
\hline
\end{tabular}

Amplitude - millivolts (motor nerves) or microvolts (sensory nerves); conduction velocity-meters/second; distal latency—milliseconds; F-wave latency-milliseconds; NR-no response; ND—not done.

bosacral spine was unrevealing. A pelvic CT demonstrated flattening of the right femoral head considered suspicious for early osteonecrosis and a bone scan revealed abnormal uptake in the right hip. Other radiologic studies, including a lumbar myelogram and a chest and lumbar spine roentenogram, were normal.

The patient continued to experience crampy pain in his left buttock, and radicular symptoms in the left leg without any resolution of his weakness. In November, he developed burning pain in both feet and difficulty walking. In early December he developed discoloration and painful swelling of the toes in the right foot, which brought him again to medical attention. One day later he suddenly developed a right foot drop and a neurologic consultation was requested.

Upon further questioning, the patient admitted to continued parasthesias on the lateral aspect of the left leg, and numbness extending to the midcalf bilaterally. His pain was exacerbated by prolonged sitting or foot elevation and improved with dangling his feet over the side of the bed. He described lower back and left hip pain that kept him awake at night. He was unable to walk without assistance due to pain and weakness.

Physical Examination. On general physical examination, he was afebrile and normotensive. There were gangrenous changes involving the toes on the

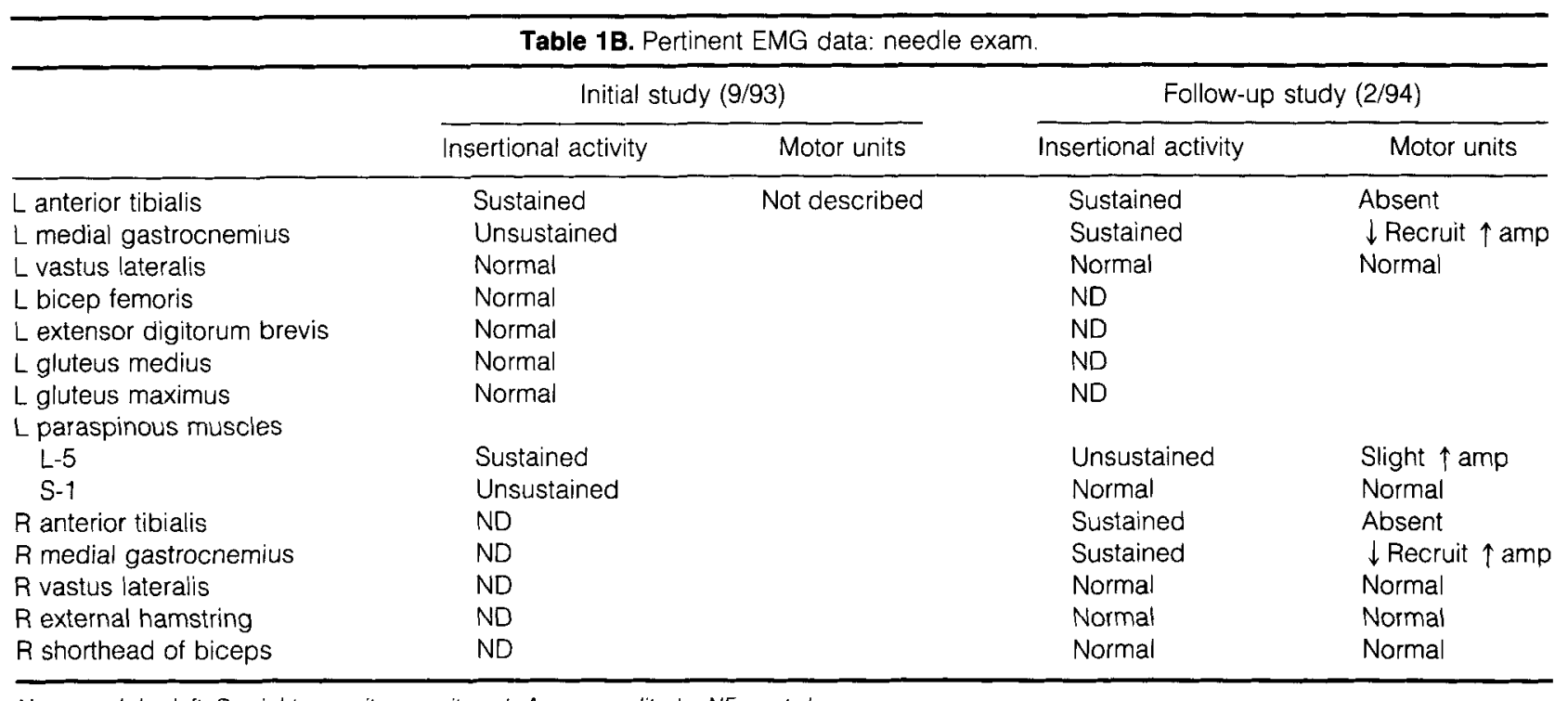

N-normal; L-veft; R-right; recruit-recruitment; Amp-amplitude; NE-not done. 
right, and bilateral foot drop (Fig. 1). Peripheral pulses were not palpable at the level of the dorsalis pedis, posterior tibial, or popliteal arteries bilaterally. Faint femoral pulse was palpable on the right. There were no carotid bruits or abnormal heart sounds.

Neurologic testing revealed a clear sensorium, intact cranial nerve function and normal upper extremity strength, sensation, and reflexes. In the lower extremities, there was distal muscular atrophy and weakness involving peroneal and tibialinnervated musculature as follows by MRC scale $(\mathrm{R} / \mathrm{L})$ : hamstrings $5 / 4+$, quadriceps $5-/ 5-$, anterior tibialis $3 / 0$, gastrocnemius $4 / 4$, ankle invertors $4 / 2$, and ankle evertors $3 / 0$. Reflexes were diminished at the left knee and absent at both ankles. Plantar responses were mute. Sensory exam demonstrated a distal gradient to pin prick, temperature, and light touch and joint position below the knee, most pronounced on the left. There was no sensory level. Sensation to pin prick was diminished proximally in the distribution of $\mathrm{L}-4$ and $\mathrm{L}-5$. Ambulation was painful, and he demonstrated a steppage gait due to bilateral foot drop.

Diagnostic Studies. On repeat evaluation, the complete blood count remained normal, while the sedimentation rate had increased to 93. Aortogram was performed the following day, demonstrating a complete occlusion of the abdominal aorta below the level of the renal arteries, with reconstitution of the external iliac and femoral arteries through the left colic and superior hemorrhoidal arteries (Fig. 2). Preoperative work-up

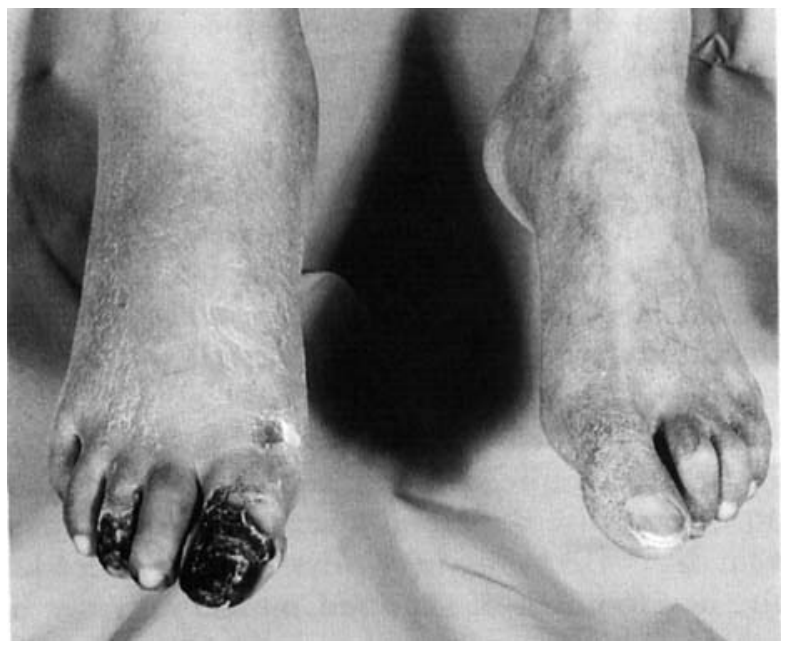

FIGURE 1. Bilateral foot drop and dry gangrene of the right foot. included a dipyridamole thallium stress test that was normal.

Postoperative Course and Follow-Up. The patient underwent urgent aortofemoral bypass grafting with removal of several organized atheromatous plaques (Fig. 3). No aneurysm or structural abnormality of the aorta was seen. The postoperative course was uncomplicated. Neurologically, his back and hip pain resolved, but he had persistent burning parathesias in both feet and continued distal weakness. Neurologic exam 2 months following surgery demonstrated continued distal weakness affecting the peroneal greater than tibialinnervated muscles graded as follows $(\mathrm{R} / \mathrm{L})$ : AT $0 / 0$, gastrocnemius $4 / 4$, ankle invertors $4 / 4$, and evertors $0 / 0$. Proximal lower extremity strength was full. Reflexes were present at the knees and absent at the ankles. Follow-up EMG demonstrated interval loss of sural sensory and tibial motor responses, and only minimal peroneal motor amplitudes on the left (Table 1). The pattern of denervation involved tibial- and peronealinnervated musculature distally, with relative sparing of proximal muscles. Isolated low amplitude spontaneous activity remained in the left lower lumbar paraspinous muscles.

\section{Discussion}

This patient provides an example of the natural history of nerve injury related to chronic large vessel occlusive disease. His presenting signs and

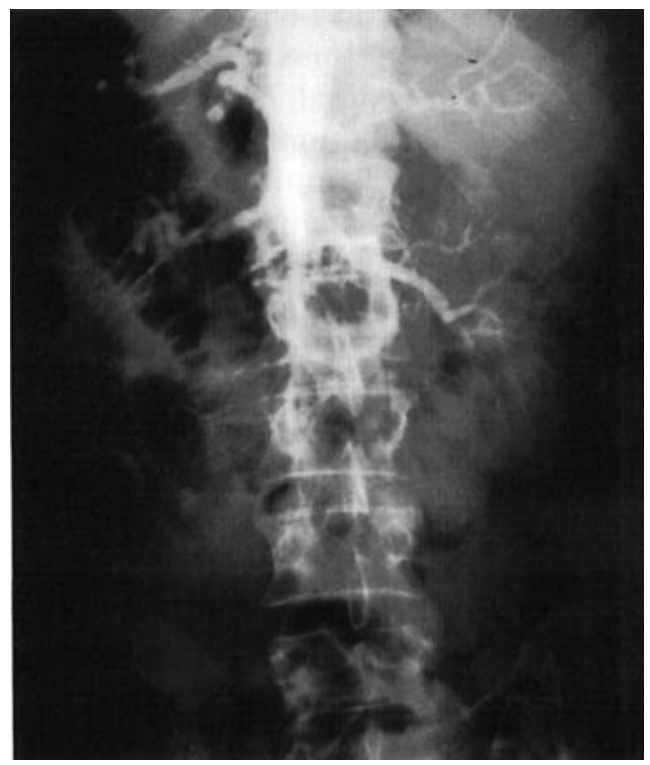

FIGURE 2. Aortogram demonstrating intrarenal occlusion of the abdominal aorta. 


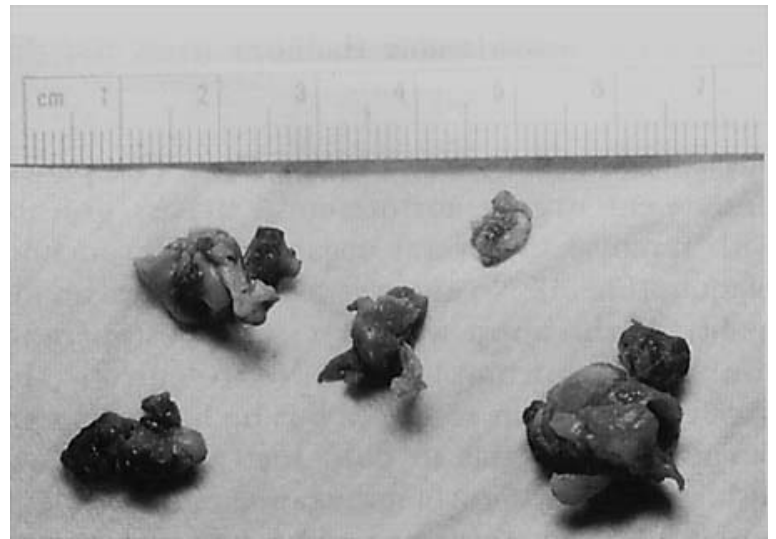

FIGURE 3. Large atheromatous plaques removed during aortofemoral bypass.

symptoms were of neurologic origin, and preceded the development of ischemic changes in the lower extremity by several months. No underlying structural lesion could be identified during the initial evaluation. The lack of concurrent evidence of peripheral vascular disease at that time may have led to the delay in his definitive diagnosis. Evaluation of leg pain frequently presents a diagnostic dilemma to both the general internist and neurologist. Potential etiologies of leg pain worsened by activity include structural spine disease, large joint disease, and peripheral arterial or venous insufficiency. ${ }^{13}$ Distinguishing clinical features of peripheral vascular insufficiency in the lower extremities include intermittent claudication with exercise, rest pain that is burning in nature, and nocturnal exacerbation of painful parasthesias likely related to low cardiac output and blood pressure. To the neurologist, these symptoms are classic for peripheral neuropathy in general, however, unremitting pain in a distal distribution can be suggestive of arterial insufficiency. Pain due to ischemia is frequently alleviated by maneuvers that increase blood flow to the extremities such as moderate activity or gravitational changes, which help distinguish it from radicular pain. ${ }^{15}$ Recognition of acute vascular insufficiency to a limb is facilitated by the accompanying changes in skin and peripheral pulses, and is frequently combined with known manipulation of the vasculature. Subacute or chronic vascular insufficiency may present in a more indolent fashion, with neuropathic pain that is hard to distinguish clinically from other more common etiologies of leg pain.

Ischemic injury to peripheral nerves is described, but is most commonly associated with acute vascular insufficiency. Determining the region of nerve injury due to large vessel disease can be difficult clinically as exemplified in our case. The location of nerve injury related to the etiology of the vascular insult, which may include a compressive phenomena from aneurysm formation, distal ischemia due to surgical manipulation of the vessel, distal embolization, or diffuse hypoperfusion as in aortic rupture. Potential sites of injury in large vessel occlusive disease, as described in previous case reports, include the root, plexus, proximal nerve at a "watershed" region, or multiple distal mononeuropathies. In our case, we consider the most likely site to be injury to the proximal sciatic nerve or lumbosacral plexus. An alternative explanation would be multifocal ischemia involving the nerve roots, in addition to the peroneal and tibial nerves distally. The patient's early symptoms of pain in the buttock with numbness extending down the leg, may have in fact represented "claudication" of the sciatic nerve, as has been described previously. ${ }^{9}$ The more pronounced clinical and electrical findings in peroneal versus tibial nerves also supports a proximal sciatic nerve localization.

The pathophysiology of nerve ischemia is not entirely defined. Most animal models of nerve function in large vessel ischemia have used the sciatic nerve of the rat. ${ }^{3,14}$ Historically, nerves were felt to be quite resistant to ischemic injury based on the structure of the vasa nervorum and extensive collateral blood supply. Early studies were problematic due to difficulty producing ischemia without concurrent compressive nerve injury. Recent studies have confirmed the presence of a "watershed" region in the distal rat sciatic nerve with increased susceptibility to vascular injury. ${ }^{8,12}$ The degree of nerve injury is also variable, depending on the duration of ischemia. Moderate ischemia has been demonstrated to produce conduction block, likely related to focal edema, whereas more prolonged ischemia many lead to axonal loss with wallerian degeneration. ${ }^{6}$ This spectrum of nerve dysfunction is supported by clinical reports of neurologic recovery following prompt revascularization after sudden vascular occlusion. ${ }^{7}$ The nerve may have a lower threshold for ischemic injury than that of skin or muscle, thus leading to neurologic deficits in the absence of visible evidence of vascular insufficiency.

Our case represents an unusual presentation of aortic occlusive disease in a relatively young man. Clinical evidence of nerve dysfunction preceded the development of skin and muscle necrosis, or other more common manifestations of subacute or chronic vascular insufficiency. This case illustrates the importance of considering aortic occlusive dis- 
ease in the evaluation of peripheral nerve injury in the lower extremities at the level of the root, plexus, or distal nerve. Early recognition of vascular insufficiency allows prompt evaluation and treatment of a potentially reversible cause of nerve dysfunction. Further characterization of the anatomic vulnerability of nerve blood supply and the pathophysiology of nerve ischemia may lead to improved recognition and understanding of this as a potentially reversible cause of nerve injury.

\section{REFERENCES}

1. Adams WE: The blood supply of nerves. / Anatomy 1942; 76:323-339.

2. D'Amour ML, Lebrun HH, Rabbat A, Trudel J, Daneault N: Peripheral neurologic complications of aorto-iliac vascular disease. Can I Neurol Sci 1987;14:127-130.

3. Day TS, Schmelzer JD, Low PA: Aortic occlusion and reperfusion and conduction, blood flow, and the bloodnerve barrier of the rat sciatic nerve. Exp Neurol 1989; 103: $173-178$.

4. Fletcher HS, Frankel J: Ruptured abdominal aneurysms presenting with unilateral peripheral neuropathy. Surgery 1976;79:120-121.

5. Glovicski P, Cross SA, Stanson AW, Carmichael SW, Bower
TC, Pairolero PC, Hallett JW, Toomey BJ, Cherry KS: Ischemic injury to the spinal cord or lumbosacral plexus after aorto-iliac reconstruction. Am J Surg 1991;162: 131-136.

6. Kaku DA, Malamut RI, Frey DJ, Parry GJ: Conduction block as an early sign of reversible nerve injury in ischemic monomelic neuropathy. Neurology 1993;43:1126-1130.

7. Kelly CJ, Augustine C, Rooney BP, Bouchier-Hayes DJ: An investigation of the pathophysiology of ischaemic neuropathy. Eur J Vasc Surg 1991;5:535-539.

8. Kinoshita Y, Monato WW: Nerve and muscle blood flow during hindlimb ischemia and reperfusion in rats. $J$ Neurosurg 1994;80:1078-1084.

9. Lamerton AJ, Bannister R, Withrington R, Seifert $\mathrm{MH}$, Eastcott HH: 'Claudication' of the sciatic nerve. $\mathrm{Br} \mathrm{Med} \mathrm{J}$ 1983;286:1785-1786.

10. Levin KH: Ischemic monomelic neuropathy. Muscle Nerve 1989;12:791-795.

11. Lynch DR, Dawson TM, Raps EC, Galetta SL: Risk factors for the neurologic complications associated with aortic aneurysms. Arch Neurol 1992;49:284-288.

12. McManis PG: Sciatic nerve lesions during cardiac surgery. Neurology 1992;44:684-687.

13. Provan JL, Moreau P, MacNab I: Pitfalls in the diagnosis of leg pain. CMA J 1979;212:167-171

14. Sladky JT, Greenberg JH, Brown MJ: Regional perfusion in normal and ischemic rat sciatic nerves. Ann Neurol 1985; $17: 191-195$.

15. Wilbourn AJ, Furlan AJ, Hulley W, Ruschhaupt W: Ischemic monomelic neuropathy. Neurology 1983;33:447-451. 